\title{
Determinação do melhor nível de sal comum para codornas japonesas em postura
}

\author{
Alice Eiko Murakami ${ }^{1}$, Márcia Izumi Sakamoto², Luciana Maria Garcia de Souza², José \\ Rodrigo Galli Francoㄹ, Mariela Akie Okino Mituo ${ }^{3}$
}

\footnotetext{
1 Departamento de Zootecnia da Universidade Estadual de Maringá /UEM/Maringá - PR.

2 Pós-Graduação em Zootecnia da Universidade Estadual de Maringá /UEM/Maringá - PR.

${ }^{3}$ Graduação - Bolsista PIBIC/CNPq da Universidade Estadual de Maringá /UEM/Maringá - PR.
}

RESUMO - Este estudo foi realizado com o objetivo de determinar o melhor nível de sal comum para codornas japonesas (Coturnix coturnix japonica) em postura. Foram utilizadas 336 codornas com 13 semanas de idade, alojadas em gaiolas de $118 \mathrm{~cm}^{2} /$ codorna durante 84 dias (quatro ciclos de 21 dias). O delineamento experimental utilizado foi inteiramente casualizado, com sete tratamentos $(0 ; 0,15 ; 0,20 ; 0,25 ; 0,30 ; 0,35$ e $0,45 \%$ de sal comum) e seis repetições de oito aves por parcela. A cada 21 dias, foram avaliados os parâmetros de desempenho (postura, consumo de ração e conversão alimentar) e qualidade dos ovos (peso médio do ovo, massa de ovo, porcentagem e espessura da casca e Unidade Haugh). Os dados obtidos foram submetidos às análises de variância e de regressão e as médias comparadas pelo teste Dunnett a 5\% de significância. A equação de regressão ajustada não foi significativa para os parâmetros avaliados em função dos níveis de sal na dieta. Entretanto, pela comparação entre as médias, observou-se que, nos tratamentos com a adição de sal, as aves apresentaram melhor desempenho produtivo e qualidade externa dos ovos, sendo que o nível de $0,15 \%$ de sal (equivalente a $0,10 \%$ de $\mathrm{Na}$ e $0,12 \%$ de $\mathrm{Cl}$ ) foi suficiente para obtenção destes resultados.

Palavras-chave: desempenho, qualidade dos ovos, umidade das excretas

\section{Determination of the best level of salt for Japanese laying quails}

\begin{abstract}
The aim of this experiment was to determine the best level of salt for Japanese laying quails (Coturnix coturnix japonica). Three hundred and thirty-six quails with 13 weeks of age were housed in cages with $118 \mathrm{~cm}^{2} /$ quail for 84 days (four cycles of 21 days each). The experiment was analyzed as a complete randomized design with seven treatments $(0$, $0.15,0.20,0.25,0.30,0.35$, and $0.45 \%$ of salt) with six replicates of eight quails per pen. Every 21 days, the productive performance (\% of production, feed intake and feed gain ratio $[\mathrm{kg} / \mathrm{kg}$ and $\mathrm{kg} / \mathrm{dozen}]$ ) and egg quality (average egg weight, egg mass, eggshell percentage and thickness and Unit Haugh) were evaluated. Data were submitted to analyses of variance and regression, and the means, compared by Dunnett test at 5\% of significance. Regression equation showed no treatment effect on productive performance. However, by the comparison among the means it was observed that the birds fed diet with salt addition showed higher values of performance and external egg quality than control (without salt). The $0.15 \%$ salt level (equivalent to $0.10 \%$ of $\mathrm{Na}$ and $0.12 \%$ of $\mathrm{Cl}$ ) was sufficient to obtain these results.
\end{abstract}

Key Words: egg quality, feces moisture, performance

\section{Introdução}

Apesar dos avanços na criação de codornas, as exigências nutricionais dessas aves ainda não estão bem definidas. As rações utilizadas nas criações são formuladas com base nas exigências nutricionais de galinhas de postura ou em dados descritos na literatura internacional, poucocondizentes com as condições brasileiras, o que compromete, às vezes, a produtividade (Murakami \& Furlan, 2002).

A composição corporal em minerais varia de 2 a $5 \%$ conforme a raça, a espécie e a própria ave. Os minerais são importantes na transferência da energia relacionada ao metabolismo celular (fósforo), na contistiuição do protoplasma e do tecido ósseo (cálcio, fósforo e magnésio) e contribuem para estabelecer e manter a pressão osmótica e o equilíbrio ácido-básico (sódio, cloro e potássio) dos animais.

$\mathrm{O}$ cloro $(\mathrm{Cl})$ é encontrado nas células, nos fluidos extracelulares do organismo, principalmente na forma de cloreto de sódio e cloreto de potássio, e no suco gástrico, como ácido clorídrico. O sódio $(\mathrm{Na})$, por sua vez, pode ser encontrado no soro sangüíneo e nos líquidos extracelulares. 
Apesar de sua importância na alimentação das aves, as exigências desses minerais têm sido pouco estudadas, talvez pelo fato de os alimentos utilizados nas rações serem ricos em potássio e as necessidades de sódio e cloro serem supridas pelo cloreto de sódio ( $\mathrm{NaCl}$ - sal comum), um ingrediente de baixo custo.

Sabe-se que deficiências de sódio na ração provocam grande redução na produção e no peso dos ovos, no consumo de ração e no peso corporal (Kuchinski et al., 1997) de galinhas de postura e, em codornas adultas, podem resultar em apatia, redução no consumo e na produtividade, baixa fecundidade e morte (Oliveira \& Almeida, 2004). Por outro lado, altos níveis de sódio na ração ocasionam au mentos significativos no consumo de água, elevação da umidade das excretas e excreção urinária de sódio, com significativa redução nas taxas de filtração glomerular (Wideman \& Buss, 1985).

Segundo Gonzales (1999), a qualidade da casca é melhorada quando se aumenta o nível de sódio e de potássio, mas piora com a elevação do nível de cloro. Na verdade, os efeitos desses íons sobre a qualidade da casca não são isolados, mas dependem do balanço entre eles, representado na equação de Mongin (1980). Contudo, segundo Austic \& Keshavarz (1984), a correlação entre o balanço eletrolítico da dieta (BED) e a espessura da casca é baixa $(r=0,2)$.

De acordo com Senkoylu et al. (2005), o aumento no BED de 176 para $242 \mathrm{mEq} / \mathrm{kg}$ não melhora a produção ou a qualidade dos ovos de galinhas poedeiras brancas. Todavia, BED de $205 \mathrm{mEq} / \mathrm{kg}$ para poedeiras no primeiro ciclo e de $174 \mathrm{mEq} / \mathrm{kg}$ para poedeiras após muda forçada têm sido eficazes em proporcionar ótima resposta produtiva e boa qualidade da casca dos ovos (Murakami et al., 2003). Além disso, segundo Reeve (1990), balanço eletrolítico superior a $190 \mathrm{mEq} / \mathrm{kg}$ seria suficiente para proporcionar adequada qualidade da casca dos ovos.

Para codornas japonesas em postura, têm sido preconizados níveis de $0,15 \%$ de $\mathrm{Na}$ e $0,11 \%$ de $\mathrm{Cl}$ na dieta (NRC, 1994) e de 0,14 e $0,18 \%$ de Na (Figueiredo et al., 2004 e Oviedo-Rondon et al., 1999, respectivamente). Em geral, os criadores de codornas formulam rações com níveis de 0,25 a $0,30 \%$ de sal comum. No entanto, são escassas as pesquisas que confirmam as recomendações desses minerais.

Desta forma, realizou-se este estudo para determinar o melhor nível de inclusão do sal comum $(\mathrm{NaCl})$ em dietas para codornas japonesas em postura.

\section{Material e Métodos}

O experimento foi conduzido no Setor de Avicultura da Fazenda Experimental de Iguatemi, da Universidade Estadual de Maringá. Foram utilizadas 336 codornas com 13 semanas de idade durante 84 dias (quatro ciclos de 21 dias). O delineamento experimental utilizado foi inteiramente casualizado em parcelas subdivididas com sete tratamentos $(0,00 ; 0,15$; 0,$20 ; 0,25 ; 0,30 ; 0,35$ e $0,45 \%$ de sal comum) e seis repetições de oito codornas. Como efeito principal, foram considerados os níveis de sal nas parcelas e, como efeito secundário, consideraram-se os ciclos (dias) nas subparcelas.

As aves foram alojadas em gaiolas sobrepostas (duas fileiras) de arame galvanizado (dimensões 38 x 25 x $14 \mathrm{~cm}$ ), com área de $118 \mathrm{~cm}^{2} /$ ave, em galpão convencional (telha de barro, mureta de alvenaria de $80 \mathrm{~cm}$ de altura e laterais completadas com tela de arame galvanizado) provido de cortinas laterais móveis. O programa de iluminação adotado constou de 17 horas de luz por dia.

As temperaturas máxima e mínima dentro do galpão foram registradas diariamente, obtendo-se médias de 29 e $19^{\circ} \mathrm{C}$, respectivamente.

As rações experimentais (Tabela 1), isonutritivas, foram formuladas à base de milho e farelo de soja, considerando-se a composição dos alimentos descrita por Rostagno et al. (2000) e as exigências nutricionais preconizadas pelo NRC (1994), exceto para os níveis de sódio e cloro.

O balanço eletrolítico das dietas (BED) experimentais foi calculado segundo Mongin (1980) utilizando-se a fórmula: $\mathrm{N}^{\circ}$ de Mongin $(\mathrm{NM})=\mathrm{mEqNa}^{+}+\mathrm{mEqK}^{+}-\mathrm{mEqCl}^{-}$ $(\mathrm{mEq} / \mathrm{kg})$.

O cálculo do NM foi realizado considerando-se os valores percentuais dos eletrólitos, por meio da seguinte fórmula: $\mathrm{NM}=\% \mathrm{Na}^{+} \times 10.000 / 22,990^{*}+\% \mathrm{~K}^{+} \times 10.000 /$ $39,102 *$ - $\% \mathrm{Cl}^{-} \times 10.000 / 35,453 *$ (* equivalente grama do $\mathrm{Na}, \mathrm{K}$ e $\mathrm{Cl}$, respectivamente).

No 21 o dia de cada ciclo, foram avaliados o consumo de ração (g/ave/dia) e a conversão alimentar ( $\mathrm{kg} / \mathrm{kg}$ e kg/dz), efetuando-se a pesagem das rações no início e ao final de cada ciclo. Os ovos foram coletados diariamente para determinação da produção de ovos (\% de postura) e a avaliação, nos últimos quatro dias de cada ciclo, do peso médio dos ovos, da altura de albúmen, da porcentagem e da espessura da casca. As cascas foram cortadas na região central, lavadas em água corrente, secas em temperatura ambiente por 48 horas e, depois de pesadas, foram utilizadas na determinação da porcentagem de casca.

A espessura da casca foi obtida com auxílio de um micrômetro rotativo manual (Mitutoyo ${ }^{\circledR}$ ) tomando-se as medidas na região central da casca, onde há maior homogeneidade da distribuição dos cristais de carbonato de cálcio - as extremidades da casca do ovo apresentam maior espessura em virtude do maior acúmulo desses cristais. 
Tabela 1 - Composição química das rações experimentais

Table 1 - Chemical composition of the experimental diets

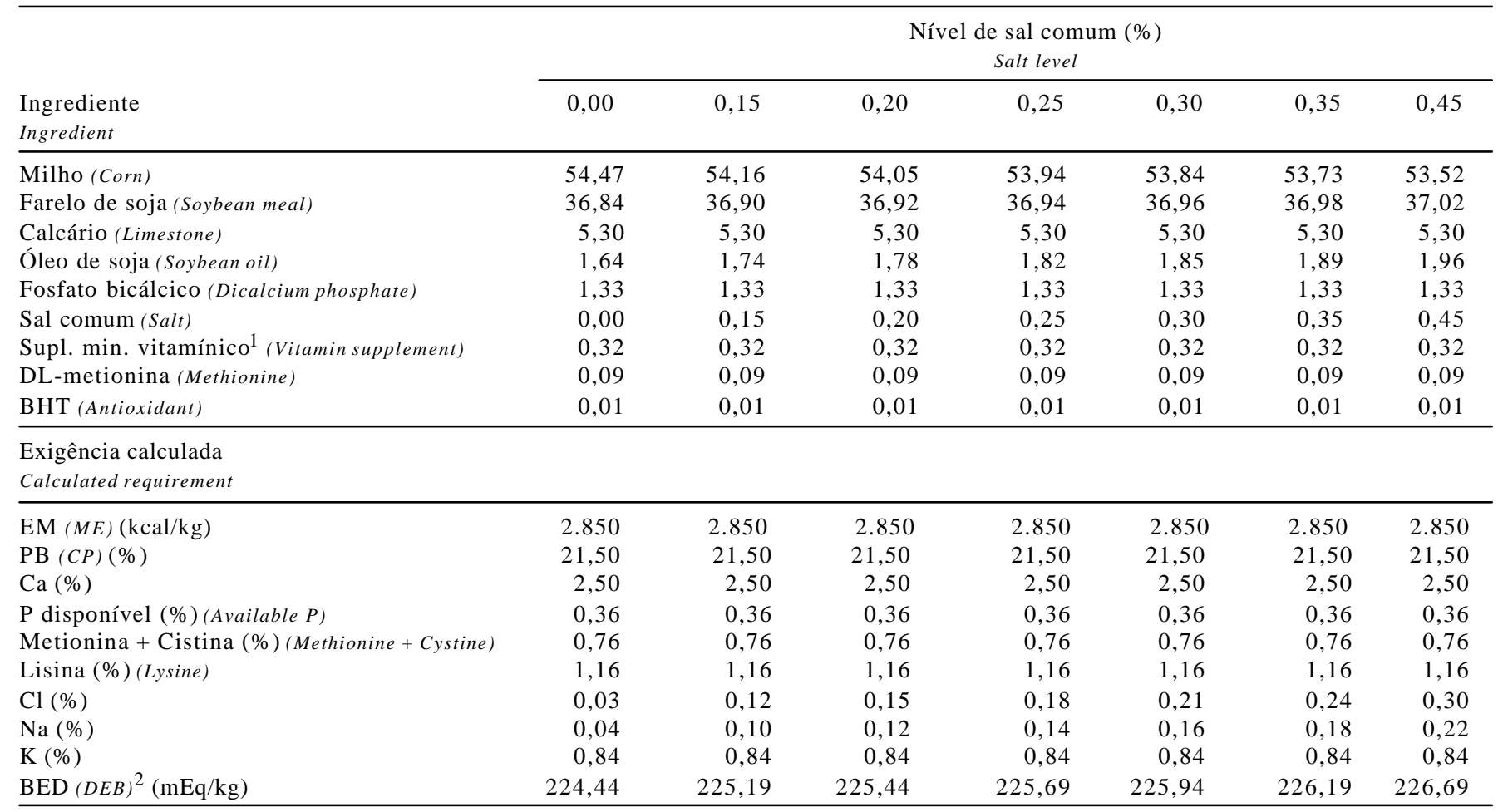

${ }^{1}$ Fornecido por kg do produto (amountperkg ofproduct): Ca - 0,213\%; Fe - 1,846\%; Co - 0,012\%; Cu - 0,373\%; Mn - 2,752\%; Zn - 1,790\%; Se - 0,006\%; I- 0,028\%; cinzas (ash) - 4,052\%; veículo (vehicle) q.s.p. - 1.000 g; vit. A - 2.586.956 UI; vit. D3 - 612.500 UI; vit. K3 - 158.261 mg; vit. B1 - 75.326 mg; vit. B2 - $973.913 \mathrm{mg}$; vit. B6 - $75.326 \mathrm{mg}$; vit. B12 - $2130.435 \mathrm{mcg}$; vit. E - $1521.739 \mathrm{mg}$; ác. pantotênico (pantothenic acid) - 2386.087 mg; niacina (niacin) - $3.766 .304 \mathrm{mg}$; vit. C - $41.384 \mathrm{mg}$; colina (choline) - $117 \mathrm{~g}$; antioxidante (antioxidant) - $913.064 \mathrm{mg} / \mathrm{kg}$; veículo (vehicle) - q.s.p.1.000 g.

2 Balanço eletrolítico da dieta (Dietary electrolyte balance).

A altura do albúmen foi obtida utilizando-se um micrômetro manual cujas mensurações, em milímetros, foram relacionadas ao peso do ovo, obtendo-se assim a Unidade Haugh, conforme descrito por Brant \& Shrader (1958): $\mathrm{UH}=100 \log \left(\mathrm{H}+7,57-1,7 \mathrm{~W}^{0,37}\right)$, em que: $\mathrm{H}=$ altura de albúmen $(\mathrm{mm}) ; \mathrm{W}=$ peso do ovo $(\mathrm{g})$.

Para avaliação da umidade das excretas, foram colocados plásticos devidamente identificados sob as gaiolas, os quais foram removidos após 8 horas de coleta de excretas. As amostras recolhidas foram pesadas e secas em estufa de ventilação forçada por 72 horas a $55^{\circ} \mathrm{C}$ (présecagem). Em seguida, foram pesadas novamente e o peso seco foi relacionado ao peso úmido para determinação da porcentagem de umidade.

Os dados obtidos para os parâmetros avaliados foram submetidos à análise de variância para os fatores nível de sal comum nas rações e períodos (ciclos) de avaliação, bem como sua interação. A análise de regressão foi utilizada para os efeitos significativos do modelo utilizando-se o programa estatístico SAEG (UFV, 1997). As médias dos tratamentos com e sem inclusão de sal às rações foram comparadas pelo teste Dunnett $(\mathrm{P}<0,05)$ pelo programa SAS $(2000)$.

\section{Resultados e Discussão}

A análise de variância comprovou efeito significativo apenas dos níveis de sal nas dietas $(\mathrm{P}<0,05)$ sobre todos os parâmetros avaliados, exceto a Unidade Haugh dos ovos e da umidade das excretas. A ausência de efeito significativo $(\mathrm{P}>0,05)$ para os períodos (ciclos) avaliados pode estar relacionada à idade das aves, pois todas se encontravam no início do pico de produção e, conseqüentemente, com grande uniformidade na produção e na qualidade dos ovos durante o período experimental.

A equação de regressão ajustada para os parâmetros avaliados em função dos níveis de inclusão de sal comum às rações não foi significativa $(\mathrm{P}>0,05)$. No entanto, pelo teste de Dunnett, foi possível observar que as aves que receberam sal na ração, em comparação às do tratamento controle, apresentaram melhor $(\mathrm{P}<0,05)$ desempenho produtivo e qualidade dos ovos, exceto para Unidade Haugh, mesmo no menor nível de inclusão $(0,15 \%$ de sal, equivalente a $0,10 \%$ de $\mathrm{Na}$ e $0,12 \%$ de $\mathrm{Cl}$ ) (Tabela 2 ).

O nível de inclusão de sal observado neste experimento $(0,15 \%)$ foi inferior aos utilizados por nutricionistas, que 
Tabela 2 - Desempenho produtivo e qualidade dos ovos de codornas japonesas alimentadas com rações contendo diferentes níveis de sal comum

Table 2 - $\quad$ Productive performance and egg quality of Japanese quails fed diets with different salt levels

\begin{tabular}{|c|c|c|c|c|c|c|c|c|}
\hline \multirow[b]{2}{*}{$\begin{array}{l}\text { Parâmetro } \\
\text { Parameter }\end{array}$} & \multicolumn{7}{|c|}{$\begin{array}{c}\text { Nível de sal comum (\%) } \\
\text { Salt level }\end{array}$} & \multirow[t]{2}{*}{$\mathrm{CV}(\%)^{2}$} \\
\hline & 0,00 & 0,15 & 0,20 & 0,25 & 0,30 & 0,35 & 0,45 & \\
\hline $\begin{array}{l}\text { Postura }(\%) \\
\text { Posture }\end{array}$ & $15,93 b$ & $81,90 \mathrm{a}$ & $81,84 \mathrm{a}$ & $75,26 a$ & $83,68 \mathrm{a}$ & $74,07 \mathrm{a}$ & $81,41 \mathrm{a}$ & 9,52 \\
\hline Consumo de ração(g/ave/dia) & $19,03 b$ & $23,71 \mathrm{a}$ & $23,82 \mathrm{a}$ & $22,15 \mathrm{a}$ & $25,93 a$ & $24,61 \mathrm{a}$ & $25,66 \mathrm{a}$ & 6,98 \\
\hline $\begin{array}{l}\text { Feed intake }(\mathrm{g} / \mathrm{bird} / \text { day }) \\
\text { Conversão alimentar }(\mathrm{kg} / \mathrm{kg})^{1} \\
\text { Feed-to-gain ratio }(\mathrm{kg} / \mathrm{kg})\end{array}$ & $11,576 \mathrm{a}$ & $2,619 b$ & $2,583 b$ & $2,605 b$ & $2,774 b$ & $2,948 b$ & $2,832 b$ & 39,96 \\
\hline $\begin{array}{l}\text { Conversão alimentar }(\mathrm{kg} / \mathrm{dz})^{1} \\
\text { Feed-to-gain ratio }(\mathrm{kg} / \text { dozen })\end{array}$ & $1,483 \mathrm{a}$ & $0,376 b$ & $0,376 \mathrm{~b}$ & $0,357 b$ & $0,355 b$ & $0,360 \mathrm{~b}$ & $0,346 b$ & 34,67 \\
\hline $\begin{array}{l}\text { Peso do ovo }(\mathrm{g}) \\
\text { Egg weight }(g)\end{array}$ & $10,32 b$ & $11,05 \mathrm{a}$ & $11,27 \mathrm{a}$ & $11,30 \mathrm{a}$ & $11,17 \mathrm{a}$ & $11,27 \mathrm{a}$ & $11,13 \mathrm{a}$ & 3,65 \\
\hline $\begin{array}{l}\text { Massa de ovo }(\mathrm{g}) \\
\operatorname{Egg} \operatorname{mass}(\mathrm{g})\end{array}$ & $1,64 b$ & $9,05 \mathrm{a}$ & $9,22 \mathrm{a}$ & $8,50 \mathrm{a}$ & $9,35 \mathrm{a}$ & $8,35 \mathrm{a}$ & $9,06 \mathrm{a}$ & 13,01 \\
\hline $\begin{array}{l}\text { Casca }(\%) \\
\text { Egg shell }(\%)\end{array}$ & $6,57 b$ & $7,65 \mathrm{a}$ & $7,69 \mathrm{a}$ & $7,71 \mathrm{a}$ & $7,61 \mathrm{a}$ & $7,71 \mathrm{a}$ & $7,83 \mathrm{a}$ & 2,51 \\
\hline $\begin{array}{l}\text { Espessura da casca }(\mathrm{mm}) \\
\text { Thickness }(\mathrm{mm})\end{array}$ & $0,208 b$ & $0,250 \mathrm{a}$ & $0,249 \mathrm{a}$ & $0,254 \mathrm{a}$ & $0,249 a$ & $0,248 \mathrm{a}$ & $0,252 \mathrm{a}$ & 5,10 \\
\hline $\begin{array}{l}\text { Unidade Haugh } \\
\text { Unit Haugh }\end{array}$ & $92,21 \mathrm{a}$ & $93,71 \mathrm{a}$ & $94,28 \mathrm{a}$ & $94,28 \mathrm{a}$ & $93,74 \mathrm{a}$ & $93,88 \mathrm{a}$ & $94,07 \mathrm{a}$ & 1,48 \\
\hline $\begin{array}{l}\text { Umiddade das excretas }(\%) \\
\text { Feces moisture }(\%)\end{array}$ & $75,36 \mathrm{a}$ & $77,14 \mathrm{a}$ & $78,07 \mathrm{a}$ & $77,85 \mathrm{a}$ & $76,50 \mathrm{a}$ & $76,66 \mathrm{a}$ & $80,33 a$ & 5,09 \\
\hline $\begin{array}{l}\text { Valor calculado } \\
\text { Calculated value }\end{array}$ & & & & & & & & \\
\hline $\begin{array}{l}\mathrm{Na}(\%) \\
\mathrm{Cl}(\%)\end{array}$ & $\begin{array}{l}0,04 \\
0,03\end{array}$ & $\begin{array}{l}0,10 \\
0,12\end{array}$ & $\begin{array}{l}0,12 \\
0,15\end{array}$ & $\begin{array}{l}0,14 \\
0,18\end{array}$ & $\begin{array}{l}0,16 \\
0,21\end{array}$ & $\begin{array}{l}0,18 \\
0,24\end{array}$ & $\begin{array}{l}0,22 \\
0,30\end{array}$ & - \\
\hline
\end{tabular}

Médias seguidas de letras diferentes na mesma linha diferem $(P<0,05)$ pelo teste Dunnett a $5 \%$.

Means followed by different letters in the same row differ $(P<0.05$ by Dunnett test.

${ }^{1} \mathrm{CA}$ - Conversão alimentar (kilograma de ração/kilograma de ovos; kilograma de ração/dúzia de ovos).

$1 \mathrm{FGR}$ - Feed-to-gain ratio ( $\mathrm{kg} / \mathrm{kg}$ and $\mathrm{kg} /$ dozen).

$2 \mathrm{CV}$ - Coeficiente de variação (\%) (Coeficient of variation, \%).

variam de 0,25 a $0,30 \%$ de $\mathrm{NaCl}$ na ração (Murakami \& Furlan, 2002).

Verificam-se controvérsias na literatura quanto aos níveis de $\mathrm{Na}$ e $\mathrm{Cl}$ para as codornas na fase de postura. $\mathrm{O}$ nível de $\mathrm{Na}$ que proporcionou o melhor resultado neste experimento $(0,10 \%)$ foi inferior ao recomendado por Shim \& Vohra (1984), de 0,12\% de Na. O NRC (1994) recomenda $0,15 \%$ de $\mathrm{Na}$ e $0,11 \%$ de $\mathrm{Cl}$, enquanto Oviedo-Rondon et al. (1999) sugeriram níveis de 0,17 a $0,18 \%$ de $\mathrm{Na}$ para melhor produtividade de codornas em postura. Da mesma forma, Figueiredo et al. (2004) recomendaram nível de $0,14 \%$ de Na para codornas em postura no verão, condições de temperaturas médias semelhantes às deste experimento. Um dos motivos para esta variação nos dados da literatura pode ser a idade das aves avaliadas, bem como as condições de manejo e instalações.

Neste estudo, observou-se que a ausência de sal (ração controle) provocou grande redução na produtivi- dade, no consumo de ração, na qualidade externa dos ovos (Tabela 2) e no peso corporal (dados não apresentados) das codornas em estudo, como observado por Oliveira \& Almeida (2004). A qualidade interna (Unidade Haugh) dos ovos, no entanto, não foi influenciada $(\mathrm{P}>0,05)$ pelos tratamentos, o que está de acordo com os resultados encontrados por Figueiredo et al. (2004), que utilizaram níveis crescentes de sódio $(0,11 ; 0,14 ; 0,17 ; 0,20$ e $0,23 \%)$ em rações para codornas em postura. Esse parâmetro de qualidade, no entanto, depende da altura do albúmen e não está relacionada ao peso do ovo, exceto após o período de armazenamento (Silversides \& Villeneuve, 1994), o que pode ter influenciado os resultados, visto que as avaliações foram feitas em ovos frescos.

A umidade das excretas também não diferiu $(\mathrm{P}>0,05)$ entre os tratamentos, indicando que os níveis de sal utilizados não provocaram consumo exagerado de água como descrito por Wideman \& Buss (1985). 


\section{Conclusões}

A inclusão de $0,15 \%$ de sal comum, equivalente a $0,10 \%$ $\mathrm{Na}$ e $0,12 \% \mathrm{Cl}$, em rações para codornas (de 13 a 25 semanas de idade) em postura foi suficiente para proporcionar bom desempenho produtivo e qualidade dos ovos.

\section{Literatura Citada}

AUSTIC, R.E.; KESHAVARZ, K. Dietary electrolytes and egg shell quality. In: CORNELL NUTRITION CONFERENCE FOR FEED MANUFACTURES, 1984, Syracuse. Proceedings... Syracuse: University of Cornell, 1984. p.63-69.

BRANT, A.W.; SHRADER, H.L. Equipment and methods for measuring egg quality. Washington: Department of Agriculture, 1958. 17p. (Agricultural Marketing Service, 246)

FIGUEIREDO, G.O.; KATO, R.K.; BERTECHINI, A.G. et al. Níveis de sódio para codornas japonesas (Coturnix coturnix japonica) no verão. In: SIMPÓSIO INTERNACIONAL, 2., CONGRESSO BRASILEIRO DE COTURNICULTURA, 1., 2004, Lavras. Anais... Lavras: Universidade Federal de Lavras, 2004. p.216.

GONZALES, E.A. [1999] Qualidade da casca do ovo. Revista Alimentação Animal, n.16, 1999. Disponível em: <http://www.bichoonline.com.br/artigos/aa0016.htm > Acesso em: 29/12/2004.

KUCHINSKI, K.K.; HARMS, R.H.; RUSSEL, G. Re-evaluation of the sodium of the commercial laying hen. In: ANNUAL MEETING POULTRY SCIENCE, 86., 1997, Lousville. Proceedings... Lousville: Poultry Science Association, 1997. v.59, p.236 (suppl. 1).

MONGIN, P. Electrolytes in nutrition: a review of basic principles and practical application in poultry and swine. In: ANNUAL MINESOTTA CONFERENCE, 3., 1980, Illinois. Proceedings... Illinois: Institute Minessota Center, 1980. p.1-15.

MURAKAMI, A.E.; FURLAN, A.C. Pesquisas na nutrição e alimentação de codornas em postura no Brasil. In: SIMPÓSIO INTERNACIONAL DE COTURNICULTURA, 1., 2002, Lavras. Anais... Lavras: Universidade Federal de Lavras, 2002. p. 113-120.
MURAKAMI, A.E.; FIGUEIREDO, D.F.; PERUZZI, A.Z. et al. Níveis de sódio para poedeiras comerciais no primeiro e segundo ciclos de produção. Revista Brasileira de Zootecnia, v.32, n.6, p.1674-1680, 2003 (supl. 1).

NATIONAL RESEARCH COUNCIL - NRC. Nutrient requirements of poultry. 9.ed. Washington: National Academic Press, 1994. 155p.

OLIVEIRA, E.G.; ALMEIDA, M.I.M. Algumas informações sobre nutrição de codornas de corte. In: SIMPÓSIO INTERNACIONAL, 2., CONGRESSO BRASILEIRO DE COTURniCUltura, 1., 2004, Lavras. Anais... Lavras: Universidade Federal de Lavras, 2004. p.53-64.

OVIEDO-RONDON, E.O.; MURAKAMI, A.E.; MORAES, E.R.G. et al. Exigência nutricional de sódio de codornas japonesas (Coturnix coturnix japonica) em postura. Revista Brasileira de Ciência Avícola, v.1, n.1, p.73-76, 1999.

REEVE, A. The role of sodium bicarbonate in improving eggshell quality. Manage Ment Guide. Lyon: Institut de Selection Animale, 1990. p.1-7 (Technical Note, 19).

ROSTAGNO, H.S.; ALBINO, L.F.T.; DONZELE, J.L. et al. Tabelas brasileiras para aves e suínos: composição de alimentos e exigências nutricionais. Viçosa, MG: Universidade Federal de Viçosa, 2000. 141p.

STATISTICAL ANALYSIS SYSTEM - SAS. SAS ${ }^{\circledR}$ user's guide: statistics. 4.ed. Version 8.1, Cary: 2000. v.2. (CD-ROM)

SENKOYLU, N.; AKYUREK, H.; SAMLI, H.E. et al. Assessment the impacts of dietary electrolyte balance levels on laying performance of commercial White layers. Pakistan Journal of Nutrition, v.4, n.6, p.423-427, 2005.

SHIM, K.F.; VOHRA, P. A review of the nutrition of Japanese quail. World Poultry Science Journal, v.40, n.3, p.261-74, 1984.

SILVERSIDES, F.G.; VILLENEUVE, P. Is the Haugh unit correction for egg weight valid for eggs stored at room temperature? Poultry Science, v.73, n.1, p.50-55, 1994.

UNIVERSIDADE FEDERAL DE VIÇOSA - UFV.SAEG - Sistema de Análises Estatísticas e Genética. Viçosa, MG: 1997. 59p.

WIDEMAN, R.F.; BUSS, E.G. Arterial blood gas, pH, and bicarbonate values in laying hens for thin eggshell production. Poultry Science, v.64, p.1015-1019, 1985. 